Review began 11/19/2021 Review ended 12/15/2021 Published 12/27/2021

(c) Copyright 2021 Alhejily. This is an open access article distributed under the terms of the Creative Commons Attribution License CC-BY 4.0., which permits unrestricted use, distribution, and reproduction in any medium, provided the original author and source are credited.

\section{Impact of the COVID-19 Pandemic on Patients With Acute Coronary Syndrome: A Tertiary Center Experience With Primary Percutaneous Intervention and Early Invasive Strategy}

\author{
Wesam Alhejily ${ }^{1,2}$ \\ 1. Cardiology, King Abdulaziz University Faculty of Medicine, Jeddah, SAU 2. Medicine, Dr. Sulaiman Al Habib Medical \\ Group, Riyadh, SAU
}

Corresponding author: Wesam Alhejily,dr.wesam.h@gmail.com

\begin{abstract}
Objective: This study aimed to determine the impact of the coronavirus disease 2019 (COVID-19) pandemic on patients with acute coronary syndrome (ACS).

Methods: This retrospective longitudinal cohort study analysed ACS patients admitted in a large tertiary center in 2019 (pre-pandemic) and 2020 (pandemic). The primary endpoint was mortality from myocardial infarction; secondary endpoints were death from any causes, including COVID-related complications, stroke, and coronary artery bypass graft surgery.
\end{abstract}

Results: There were 489 ACS patients admitted in 2020, and 614 in 2019, representing a 21\% reduction $(p=0.001)$. Male patients comprised $73 \%$ of the patients. Only eight were polymerase chain reaction (PCR)confirmed positive COVID-19 patients. The mean time to presentation from the time of onset of symptoms in acute ST-elevation myocardial infarction cases was $48 \pm 16$ in 2020 (vs. $4 \pm 3$ h in 2019); this significant delay was observed in more than $50 \%$ of patients ( $\mathrm{p}=0.0001$ ). Mortality due to ACS in 2020 doubled, with eight patients confirmed dead during or within 30 days of admission, with none of the deaths related to COVID-19. The incidence of stroke $(\mathrm{p}=0.01)$ and coronary artery $(\mathrm{p}=0.0001)$ bypass was also high in 2020 .

Conclusion: We found a statistically significant increase in the mortality related to myocardial infarction. Despite timely interventions, patients presented late and were worse than in the non-pandemic period.

Categories: Cardiology

Keywords: pandemic, outcomes, time, myocardial infarction, covid-19

\section{Introduction}

The novel coronavirus disease 2019 (COVID-19), which can lead to acute respiratory distress syndrome, was first reported in December 2019 in Wuhan, China. Owing to its vast and rapid spread across the globe, the World Health Organization declared this global health emergency a pandemic on March 11, 2020 [1]. By December 2020, more than 80 million COVID-19 cases were reported, accounting for approximately two million direct deaths $[2,3]$. The number of acute coronary syndrome (ACS) cases typically increases during the flu season due to an increase in inflammatory markers leading to atherosclerotic plaque instability, which was confirmed in the autopsy of patients dying from common flu $[4,5]$.

In a survey conducted by Pessoa-Amorim et al. across many hospitals worldwide, $78.8 \%$ of the participants responded that the number of hospital admissions of patients presenting with acute ST-elevation myocardial infarction (STEMI) decreased by more than $40 \%$ after the coronavirus outbreak [6]. Most participants reported that STEMI patients presented later than usual, and that $>40 \%$ of STEMI patients admitted to hospital presented beyond the optimal window for primary percutaneous intervention (PCI) or thrombolysis. Predictors of delayed STEMI presentation included a country in total lockdown, >100 COVID19 cases admitted locally, and the complete restructuring of the local cardiology service. Any delay in treatment related to acute ST-elevation myocardial infarction (MI) affects the patients' life and outcomes, particularly in those severely ill with cardiogenic shock. Scholz et al. found that every 10-minute delay in treatment resulted in 3.31 additional deaths per 100 PCI-treated patients [7].

Saudi Arabia has enforced several strict measures to contain the spread of the infection. For example, the seasonal Umrah and Pilgrimage for local and international worshippers were suspended. Malls and public places, such as parks, mosques, restaurants, and cafes, were closed, and classes were suspended. A shift to work from home was also adopted. In addition, a complete lockdown and partial and complete curfew were imposed several times to halt the spread of the virus. International and domestic travel was also restricted. These measures led to greater containment and control of the virus in Saudi Arabia compared with other 
countries where the number of infections has already spiraled out of control. The first confirmed case of COVID-19 infection in Saudi Arabia was reported on March 22, 2020. By December 31, the total number of cases reached more than 300,000, and about 6000 deaths were reported [8]. Public awareness campaigns were amplified on Ministry of Health (MOH) websites and different social media platforms. Daily press conferences with updates on the number of new confirmed cases and deaths were conducted, highlighting the possible causes of some case clusters. Patients from different backgrounds were guided using a toll-free hotline of the MOH. Regarding the healthcare setting, elective surgical operations were put on hold, appointments were rescheduled, and hospitals were mainly dedicated to treating emergency cases and patients with complicated COVID-19. Many field hospitals were also constructed in anticipation of patient overflow.

Despite the stress on the timely presentation of MI, we noticed a dramatic decline in the number of MI cases, particularly when curfew was in effect. Thus, in this prospective cohort study, we analysed the data of patients with ACS who were diagnosed during the pandemic, namely 2020, and compared their outcomes with those diagnosed in 2019. We aimed to assess the indirect impact of the pandemic on ACS patients.

\section{Materials And Methods}

The characteristics and clinical data of all patients with ACS during the COVID-19 pandemic were collected and compared with those from the same months of the previous year; data were gathered using the health information system database repository, in addition to catheterization laboratories databank in a large tertiary hospital with primary PCI capabilities. Mortality and surrogate endpoints, such as time from the onset of symptoms to presentation, door-to-balloon time and ejection fraction, were analysed. The primary endpoint was mortality related directly to ACS. The secondary endpoint was the composite of death from any cause, including COVID-19 infection, recurrent MI, or heart failure within 30 days. Analysis of variance (ANOVA) was used to examine the differences in variance across the means. Two-tailed tests were performed, and statistical significance was set at $\mathrm{p}=0.05$. Patient characteristics and risk factors were compared. Other parameters, such as the need for emergency coronary artery bypass graft (CABG) surgery and the incidence of acute stroke, were also studied. Results were tabulated and graphed for comparison; data, including age and door-to-balloon time, are presented as means \pm standard deviations. The study protocol was approved by the institutional review board research committee, with access to the database. All patient-related information was anonymized and was Health Insurance Portability and Accountability Act compliant. ANOVA was used as we had categorical independent variables like death, stroke and CABG (with two or more categories); a paired t-test was used for continuous variables like time. All computations were made using IBM SPSS Statistics, version 22 (IBM Corp., Armonk, NY).

\section{Results}

This retrospective cohort analysis of patients with ACS was conducted from January 2020 to December 31, 2020 . We noted a decline of $17 \%$ in cardiac catheterization laboratories cases during the curfew time from early March to mid-May 2020 (Figure 1).

\section{Grand Total Cath Lab Procedures}

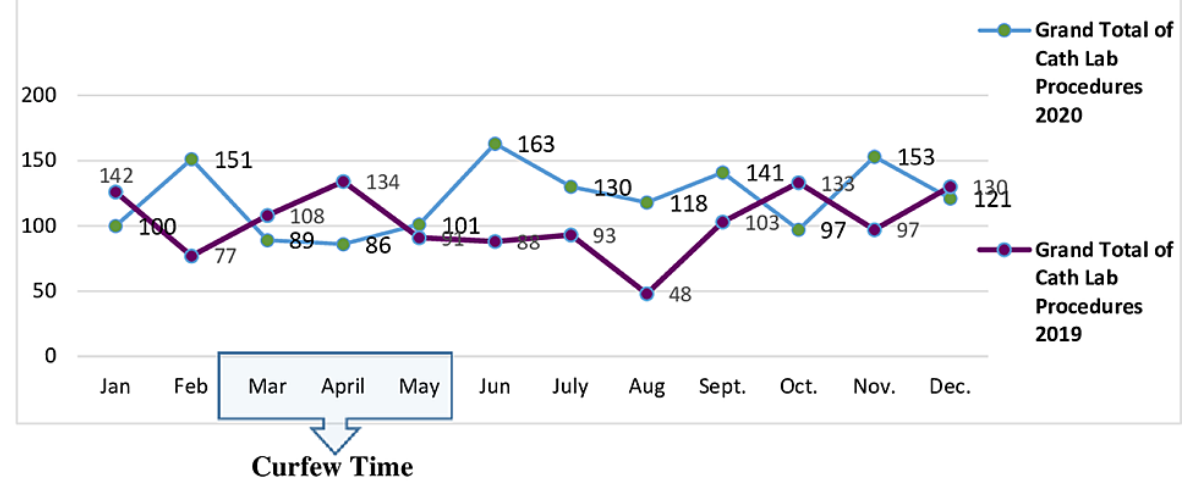

FIGURE 1: Monthly number of catheterization laboratory procedures in 2019 (violet line) and 2020 (blue line). Months when curfew was implemented are enclosed in a rectangle.

We identified 489 ACS cases in 2020 and 614 cases in the year prior to the pandemic. All patients were admitted to hospitals, with a $21 \%$ reduction in the number of admissions. Of these, 357 patients were male, representing $73 \%$ of the population. The dominant risk factors were hypertension (53\%), diabetes (51\%), and 


\section{Cureus}

hypercholesteremia (5\%). Risk factors were comparable (Table 1).

\begin{tabular}{|c|c|c|c|}
\hline ACS patients' characteristics & 2019 (pre-COVID-19) & 2020 (during COVID-19) & p value ${ }^{*}$ \\
\hline Total number & $\mathrm{N}=614$ & $N=489$ & $0.001^{*}$ \\
\hline Mean age & $53 \pm 7$ & $55 \pm 7$ & 0.4 \\
\hline Gender (male/female) & $319 / 295$ & $357 / 132$ & 0.15 \\
\hline Nationality (Saudi/non-Saudi) & $465 / 149$ & $376 / 113$ & 0.59 \\
\hline Hypertension & 235 & 260 & 0.8 \\
\hline Diabetes & 217 & 249 & 0.7 \\
\hline Smoking & 70 & 30 & $0.046^{*}$ \\
\hline Need for $C A B G$ & 9 & 26 & 0.032 \\
\hline Atrial fibrillation & 14 & 12 & 0.06 \\
\hline Renal Impairment & 56 & 69 & 0.08 \\
\hline Hyperlipidemia & 57 & 24 & 0.05 \\
\hline Mean ejection fraction & $50 \pm 5$ & $48 \pm 5$ & 0.9 \\
\hline Time to presentation & $4 \pm 3$ hours & $48 \pm 16$ hours & $0.0001^{*}$ \\
\hline
\end{tabular}

\section{TABLE 1: Characteristics of patients with ACS}

ACS, acute coronary syndrome; COVID-19, coronavirus disease 2019; CABG, coronary artery bypass graft

*Statistically significant $p<0.05$

Only eight cases of reverse transcriptase-polymerase chain reaction (RT-PCR)-confirmed COVID-19 infection were related to an acute coronary event, despite a total number of 6000 confirmed COVID-19 cases. There were six non-STEMI cases; the delay to catheterization was related to the time needed for result confirmation, which took 24 hours. Only two of these cases were STEMI and were taken to the catheterization laboratory without delay. Swabs were collected en route to the cardiac catheterization laboratory, and diagnosis was made after primary PCI was performed. Visual triage using a questionnaire was conducted for all those coming to the hospital, along with temperature measurement, and this may have contributed to some delays in starting cases in the catheterization laboratories. Patients identified as high risk were swabbed by trained staff in full personal protection equipment. As we applied the primary PCI strategy to all coming with STEMI and an early invasive strategy to all other ACS categories within 4-24 hours, a zero-infection rate among the catheterization laboratory team was reported.

The mean time to presentation from the onset of symptoms in STEMI cases was $48^{ \pm} 16$ hours compared to $4 \pm 3$ hours with a significant delay during the COVID-19 pandemic in more than $50 \%$ of the patients $(\mathrm{p}=0.0001)$. However, the mean door-to-balloon time was 89.91 and 106.14 minutes for patients presenting directly and indirectly to the hospital; for patients who presented to other facilities, the time was calculated from the first medical contact to balloon deployment instead of time from door to balloon. Times were higher than the time of the last year of 76.54 and 102.31 minutes, but still within the 90- and 120minute range, respectively This was mainly related to more strict measures related to traffics and triaging patients and medical staff attending hospitals. The mean ejection fraction was $48 \pm 5 \%$, lower than the last year before the COVID-19 pandemic $50 \pm 5 \%$. Mortality due to ACS doubled during the pandemic, with eight patients confirmed dead; seven of these cases were related to arrhythmogenic death at presentation and one was related to ruptured ventricular septum that was discovered after 14 days of hospital discharge, versus only four cases in the year earlier. This was despite the primary PCI policy being applied to all those coming, with no significant difference in door-to-balloon time for STEMI cases. Since there was no direct death related to COVID-19, all-cause mortality and death due to ACS were similar (Table 2). 


\section{Cureus}

\begin{tabular}{|c|c|c|c|}
\hline Classification & 2019 & 2020 & $p$ value \\
\hline STEMI & $55(8.9 \%)$ & $42(8.6 \%)$ & 0.89 \\
\hline Mean door-to-balloon time (direct) & $76.54 \mathrm{~min}$ & $89.91 \mathrm{~min}$ & 0.68 \\
\hline Mean first medical contact to balloon time & $102.31 \mathrm{~min}$ & $106.14 \mathrm{~min}$ & 0.75 \\
\hline NSTEMI & $16(2.6 \%)$ & $28(5.7 \%)$ & $0.046^{*}$ \\
\hline Unstable angina & $543(88 \%)$ & $419(86 \%)$ & 0.78 \\
\hline Use of intra-aortic balloon pump & $10(1.6 \%)$ & $1(0.2 \%)$ & $0.001^{*}$ \\
\hline Use of inotropic support & $8(1.3 \%)$ & $2(0.4 \%)$ & $0.01^{*}$ \\
\hline COVID-19-positive status & $0(0 \%)$ & $8(1.6 \%)$ & $0.04^{*}$ \\
\hline
\end{tabular}

TABLE 2: Classification of ACS patients admitted during COVID-19 and pre-COVID-19

STEMI, ST-elevation myocardial infarction; ACS, acute coronary syndrome; COVID-19, coronavirus disease 2019; NSTEMI, non-ST-elevation myocardial infarction

*Statistically significant $p<0.05$

With regard to major adverse outcomes, the rate of re-infarction (defined by a new rise in biomarkers with clinical symptoms of ACS within 30 days of discharge) was insignificant between both years. There was a trend of a higher rate of heart failure, defined by the presence of congestive symptoms, with an elevated prohormone of brain natriuretic peptide (pro BNP) level and a reduced ejection fraction of $35 \%$ or less either on initial presentation ( 23 cases representing $80 \%$ ) or on readmission within 30 days after discharge (6 cases representing 20\%), and it was statistically insignificant. Cases were managed with optimal medical therapy following international guidelines, after initial diuresis. Patients were placed on maximally tolerated therapy of angiotensin receptor neprilysin inhibitors (ARNIs), beta blockers, aldosterone antagonists, and sodium-glucose co-transporter 2 (SGLT2) inhibitors in addition to anti-ischemic therapy and anticoagulation as needed. For patients with renal failure or hyperkalemia, hydralazine and long-acting nitrate were used to substitute ARNIs and aldosterone antagonists. In contrast, there was a major increase in the rate of stoke with four cases documented to have had stroke, three during admission and one after 30 days of initial diagnosis compared to none from the year before the pandemic $(p=0.001)$; the need for urgent coronary artery bypass surgery (CABG) was higher in the COVID year, with 24 versus 9 cases with a threefold increase $(p=0.001)$. Interestingly, there was more usage of intra-aortic balloon pump (IABP) in the year prior to the pandemic likely explained by the fact that those patients presented with features of pump failure were past the window of revascularization and that they were not hemodynamically compromised; on the other hand, most deaths related to ACS were before patients were physically present in the catheterization laboratory precluding any interventions (Table 3). 


\section{Cureus}

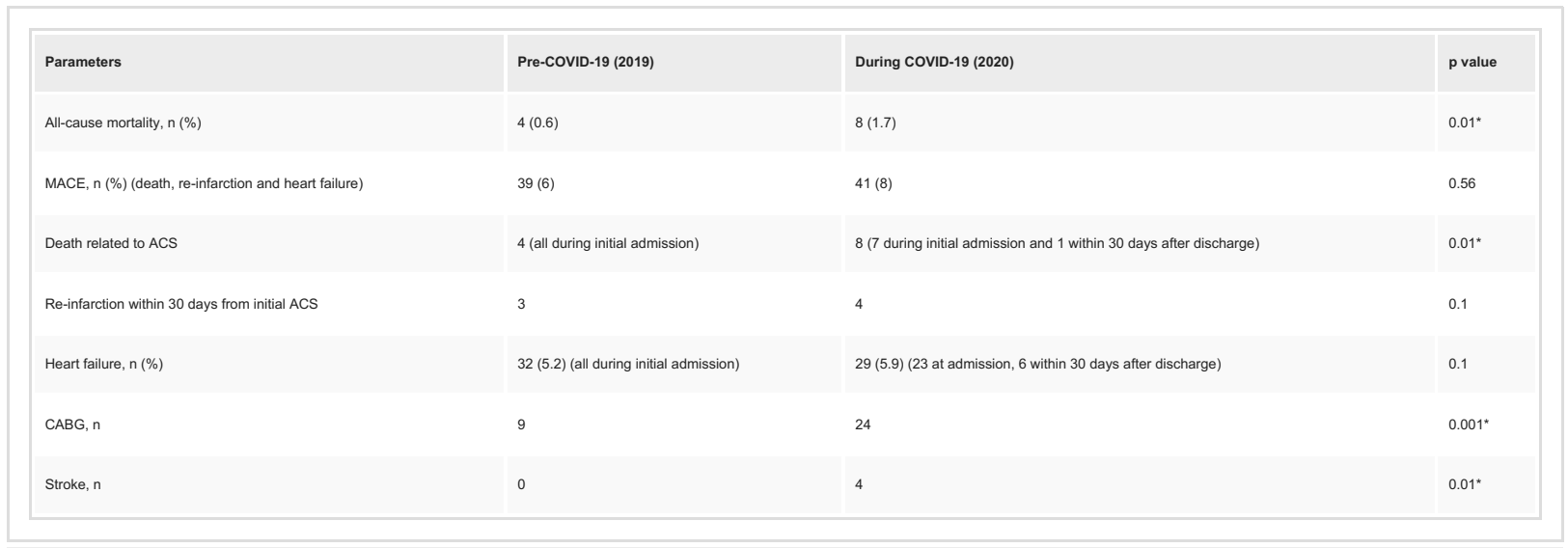

\section{TABLE 3: Mortality, MACE, CABG and stroke in different ACS populations}

MACE, major adverse cardiac events; ACS, acute coronary syndrome; COVID-19, coronavirus disease 2019; CABG, coronary artery bypass graft

*Significant $p$ value $<0.05$

\section{Discussion}

This study addressed the difference in outcomes for ACS related to the COVID-19 pandemic in the Kingdom of Saudi Arabia. Saudi Arabia has vast experience in responding to communicable diseases, which was gained from dealing with the Middle East respiratory syndrome during Hajj (pilgrimage), where more than 2.5 million worshippers from all over the world gathered for five days $[9,10]$.

In the first three months of curfew implementation, we noted a decline in ACS cases, and the total number of cases performed in the cardiac catheterization laboratories. We speculate that this is likely because of the fear of going to hospitals because of the high rate of COVID-19 cases. In our hospital, 6000 confirmed cases was reported during the first 12 months of the pandemic; more than 800 cases were admitted, and more than 50 cases of mortality were reported. When the curfew was lifted, there was an increase in the number of ACS patients, and more patients presented with ACS, including late-presentation STEMI cases (Figure 1). In addition, the number of total procedures performed decreased by $17 \%$, and ACS cases presented to us decreased by $21 \%$ compared to the pre-pandemic year 2019. As noted in the previous section, the number of deaths in 2020 remained low at $1.6 \%(n=8)$. One patient was pronounced dead in the catheterization lab, whereas most patients died within 4-30 days, either during hospitalization or after discharge. The number of deaths in 2020 was significantly higher than that in 2019 , which had a death rate of $<0.6 \%$. Re-infarction was defined as a second presentation with a new rise in troponin and a clinical picture of ACS; this was documented in three versus four cases in the pre-pandemic compared to the pandemic year. The impact of having early invasive strategy for all coming during the pandemic might have played a major role in reducing this number compared to previous series, which showed a higher rate of mortality reaching more than 20\% [11-21]. The cases seen during the pandemic had more established mechanical complications like ruptured ventricular septum and acute mitral regurgitation, but still dominated by pump failure and poor ejection fraction; left main and more diffuse three-vessel coronary artery diseases were more prevalent as the rate of bypass surgery was higher. Although the mean ejection fraction was comparable between both groups, the number of cases of heart failure was numerically higher during the pandemic time, $5.9 \%$ versus $5.2 \%$, respectively, but statistically insignificant; this was directly related to the delay from the time of onset of symptoms, which was over two days compared to five to six hours with other patients in the non-COVID period.

Regarding major adverse outcomes, we noted an increase in the overall rate of thromboembolic stroke cases coming to the hospital, particularly among ACS patients. We noted four patients with ACS who had stroke following their admission, which was likely a thromboembolic event and related to cardiac sources of embolism. Particularly, three patients had atrial fibrillation, left ventricular intramural thrombus due to poor ejection fraction, and one patient had no identifiable source of embolus. The door-to-balloon time for patients arriving directly to the emergency department or transferred from other hospitals was longer during the pandemic than during the pre-pandemic period, ranging from 90 to 120 minutes. We also noted a lower use of IABP and ionotropic support among ACS patients during the pandemic than during the pre-pandemic time. This is likely related to initial stable hemodynamics, or early death as most patients would have developed complications from MI prior to the admission period, leading to their demise and precluding any interventions.

This study had some limitations. First, this was a single-center study. Second, only a low prevalence of COVID-19 cases among ACS patients was observed. Despite these limitations, this retrospective analysis 
study provides valuable information on ACS patients during the COVID 19-pandemic. We assessed the indirect impact of the pandemic on such vulnerable patients, and the delay in their presentation that may have deleterious effects on their lives, thus highlighting the importance of health education of the public [22-30]. Public awareness campaigns highlighting the seriousness of these symptoms may play a role in ameliorating this effect. Future studies should be conducted to examine the long-term impact of the pandemic on the study population and the effect of public awareness campaigns on ACS-related symptoms and the need for timely presentation to healthcare facilities.

\section{Conclusions}

This retrospective cohort study revealed the indirect effect of the COVID-19 pandemic on patients with ACS. We noted a higher number of deaths, complications, and the need for urgent CABG and stroke. Despite the early invasive strategy, mortality was related to a delay in presentation from the time of onset of symptoms.

\section{Additional Information \\ Disclosures}

Human subjects: Consent was obtained or waived by all participants in this study. Dr. Sulaiman Al Habib Medical Group issued approval HAP-01-R-082. Animal subjects: All authors have confirmed that this study did not involve animal subjects or tissue. Conflicts of interest: In compliance with the ICMJE uniform disclosure form, all authors declare the following: Payment/services info: All authors have declared that no financial support was received from any organization for the submitted work. Financial relationships: All authors have declared that they have no financial relationships at present or within the previous three years with any organizations that might have an interest in the submitted work. Other relationships: All authors have declared that there are no other relationships or activities that could appear to have influenced the submitted work.

\section{References}

1. Virtual press conference on COVID-19 - 11 March 2020 . (2020). Accessed: March 30, 2021: https://www.who.int/docs/default-source/coronaviruse/transcripts/who-audio-emergencies-coronaviruspress-conference-f....

2. Coronavirus resource center: COVID-19 dashboard . (2020). Accessed: March 30, 2021: http://coronavirus.jhu.edu/map.html.

3. COVID-19. (2021). Accessed: March 30, 2021: https://www.cdc.gov/coronavirus/2019-ncov/index.html.

4. Warren-Gash C, Blackburn R, Whitaker H, McMenamin J, Hayward AC: Laboratory-confirmed respiratory infections as triggers for acute myocardial infarction and stroke: a self-controlled case series analysis of national linked datasets from Scotland. Eur Respir J. 2018, 51:1701794. 10.1183/13993003.01794-2017

5. Madjid M, Miller CC, Zarubaev VV, et al.: Influenza epidemics and acute respiratory disease activity are associated with a surge in autopsy-confirmed coronary heart disease death: results from 8 years of autopsies in 34,892 subjects. Eur Heart J. 2007, 28:1205-10. 10.1093/eurheartj/ehm035

6. Pessoa-Amorim G, Camm CF, Gajendragadkar P, et al.: Admission of patients with STEMI since the outbreak of the COVID-19 pandemic: a survey by the European Society of Cardiology. Eur Heart J Qual Care Clin Outcomes. 2020, 6:210-6. 10.1093/ehjqcco/qcaa046

7. Scholz KH, Maier SK, Maier LS, et al.: Impact of treatment delay on mortality in ST-segment elevation myocardial infarction (STEMI) patients presenting with and without haemodynamic instability: results from the German prospective, multicentre FITT-STEMI trial. Eur Heart J. 2018, 39:1065-74. 10.1093/eurheartj/ehy004

8. Algaissi AA, Alharbi NK, Hassanain M, Hashem AM: Preparedness and response to COVID-19 in Saudi Arabia: building on MERS experience. J Infect Public Health. 2020, 13:834-8. 10.1016/j.jiph.2020.04.016

9. Memish ZA, Perlman S, Van Kerkhove MD, Zumla A: Middle East respiratory syndrome. Lancet. 2020, 395:1063-77. 10.1016/S0140-6736(19)33221-0

10. Perrin N, Iglesias JF, Rey F, et al.: Impact of the COVID-19 pandemic on acute coronary syndromes . Swiss Med Wkly. 2020, 150:w20448. 10.4414/smw.2020.20448

11. Fileti L, Vecchio S, Moretti C, et al.: Impact of the COVID-19 pandemic on coronary invasive procedures at two Italian high-volume referral centers. J Cardiovasc Med (Hagerstown). 2020, 21:869-73. 10.2459/JCM.0000000000001101

12. Araiza-Garaygordobil D, Montalto C, Martinez-Amezcua P, et al.: Impact of the COVID-19 pandemic on hospitalizations for acute coronary syndromes: a multinational study. QJM. 2021, 114:642-7. 10.1093/qjmed/hcab013

13. Hauguel-Moreau M, Pillière R, Prati G, et al.: Impact of coronavirus disease 2019 outbreak on acute coronary syndrome admissions: four weeks to reverse the trend. J Thromb Thrombolysis. 2021, 51:31-2. 10.1007/s11239-020-02201-9

14. D'Ascenzo F, De Filippo O, Borin A, et al.: Impact of COVID-19 pandemic and infection on in hospital survival for patients presenting with acute coronary syndromes: a multicenter registry. Int J Cardiol. 2021, 332:227-34. 10.1016/j.ijcard.2021.03.063

15. Kapoor A, Yadav R: Will the hidden specter of acute coronary syndrome (ACS) and ST-segment elevation myocardial infarction (STEMI) emerge from the avalanche of COVID-19?. Indian Heart J. 2020, 72:192-3. 10.1016/j.ihj.2020.05.017

16. Vacanti G, Bramlage P, Schymik G, Schmitt C, Luik A, Swojanowsky P, Tzamalis P: Reduced rate of admissions for acute coronary syndromes during the COVID-19 pandemic: an observational analysis from a tertiary hospital in Germany. Herz. 2020, 45:663-7. 10.1007/s00059-020-04991-3 
17. Petrović M, Milovančev A, Kovačević M, Miljković T, Ilić A, Stojšić-Milosavljević A, Golubović M: Impact of COVID-19 outbreak on hospital admissions and outcome of acute coronary syndromes in a single highvolume centre in southeastern Europe. Neth Heart J. 2021, 29:230-6. 10.1007/s12471-021-01554-X

18. Haddad K, Potter BJ, Matteau A, Gobeil F, Mansour S: Implications of COVID-19 on time-sensitive STEMI care: a report from a North American epicenter. Cardiovasc Revasc Med. 2021, 30:33-7. 10.1016/j.carrev.2020.09.024

19. Vecchio S, Fileti L, Reggi A, Moschini C, Lorenzetti S, Rubboli A: Impact of the COVID-19 pandemic on admissions for acute coronary syndrome: review of the literature and single-center experience. (Article in Italian). G Ital Cardiol (Rome). 2020, 21:502-8. 10.1714/3386.33635

20. Tam CF, Cheung KS, Lam S, et al.: Impact of coronavirus disease 2019 (COVID-19) outbreak on outcome of myocardial infarction in Hong Kong, China. Catheter Cardiovasc Interv. 2021, 97:E194-7. 10.1002/ccd.28943

21. Kiss P, Carcel C, Hockham C, Peters SA: The impact of the COVID-19 pandemic on the care and management of patients with acute cardiovascular disease: a systematic review. Eur Heart J Qual Care Clin Outcomes. 2021, 7:18-27. 10.1093/ehjqcco/qcaa084

22. Braiteh N, Rehman WU, Alom M, et al.: Decrease in acute coronary syndrome presentations during the COVID-19 pandemic in upstate New York. Am Heart J. 2020, 226:147-51. 10.1016/j.ahj.2020.05.009

23. De Filippo O, D'Ascenzo F, Angelini F, et al.: Reduced rate of hospital admissions for ACS during COVID-19 outbreak in Northern Italy. N Engl J Med. 2020, 383:88-9. 10.1056/NEJMc2009166

24. Zitelny E, Newman N, Zhao D: STEMI during the COVID-19 pandemic - an evaluation of incidence . Cardiovasc Pathol. 2020, 48:107232. 10.1016/j.carpath.2020.107232

25. Xiang D, Xiang X, Zhang W, et al.: Management and outcomes of patients with STEMI during the COVID19 pandemic in China. J Am Coll Cardiol. 2020, 76:1318-24. 10.1016/j.jacc.2020.06.039

26. De Rosa S, Spaccarotella C, Basso C, et al.: Reduction of hospitalizations for myocardial infarction in Italy in the COVID-19 era. Eur Heart J. 2020, 41:2083-8. 10.1093/eurheartj/ehaa409

27. Wang N, Zhang M, Su H, Huang Z, Lin Y, Zhang M: Fibrinolysis is a reasonable alternative for STEMI care during the COVID-19 pandemic. J Int Med Res. 2020, 48:300060520966151. 10.1177/0300060520966151

28. Mahmud E, Dauerman HL, Welt FG, et al.: Management of acute myocardial infarction during the COVID-19 pandemic: a position statement from the Society for Cardiovascular Angiography and Interventions (SCAI), the American College of Cardiology (ACC), and the American College of Emergency Physicians (ACEP). J Am Coll Cardiol. 2020, 76:1375-84. 10.1016/j.jacc.2020.04.039

29. Yerasi C, Case BC, Forrestal BJ, et al.: Treatment of ST-segment elevation myocardial infarction during COVID-19 pandemic. Cardiovasc Revasc Med. 2020, 21:1024-9. 10.1016/j.carrev.2020.05.027

30. Guzik TJ, Mohiddin SA, Dimarco A, et al.: COVID-19 and the cardiovascular system: implications for risk assessment, diagnosis, and treatment options. Cardiovasc Res. 2020, 116:1666-87. 10.1093/cvr/cvaa106 